\title{
ON THE EXISTENCE AND BOUNDARY BEHAVIOR OF SOLUTIONS TO A CLASS OF NONLINEAR DIRICHLET PROBLEMS
}

\author{
CARLOS E. KENIG AND WEI-MING NI ${ }^{1}$
}

\begin{abstract}
In this paper, we first extend the well-known method of super- and sub-solutions for elliptic boundary value problems to $L^{\infty}$-boundary functions. Then we apply this method to investigate the solvability and the boundary behavior of solutions to some nonlinear elliptic equations, some Fatou-type results are obtained.
\end{abstract}

Let $L=\sum_{i, j=1}^{n} \partial_{x_{i}}\left(a_{i j}(x) \partial_{x_{j}}\right)$, where $a_{i j}(x)=a_{j i}(x) \in C^{\infty}\left(\mathbf{R}^{n}\right)$, and $\sum_{i, j} a_{i j}(x) \xi_{i} \xi_{j}$ $\geqslant \lambda|\xi|^{2}$. Let $\Omega$ be a bounded, smooth domain in $\mathbf{R}^{n}$, and $f(x, u)$ either Lipschitz in $u$ (and $C^{\alpha}$ in both variables) or increasing in $u$ (and $C^{\alpha}$ in both variables), which satisfies $f(x, u)=O\left(|u|^{p}\right)$ at $u=0$, for some $p>1$, uniformly in $x$. Our main interest in this note is to study the Dirichlet problem for the operator $L u+f(x, u)$ in $\Omega$, with boundary data $g \in L^{\infty}(\partial \Omega)$. We accomplish this by extending to our setting the classical method of super- and sub-solutions. This method goes back to Bieberbach (see the last paragraph of $[\mathbf{K}, \mathbf{W}]$ for further historical comments). More recently, this method was used in [S], in a manner very similar to ours.

Finally, the results on the boundary behavior of solutions that we obtain are identical to (and follow from) those in the linear theory, as in [ $\mathbf{W}$ or $\mathbf{J}, \mathbf{K}]$.

THEOREM 1. There exists a number $\varepsilon_{0}>0$ such that, if $\|g\|_{L^{\infty}(\partial \Omega)} \leqslant \varepsilon_{0}$, then there exists a function $u$ in $C^{2, \alpha}(\Omega)$, which satisfies $L u+f(x, u)=0$ in $\Omega$, and such that for a.e. $Q(d \sigma)$ on $\partial \Omega, u(x)$ converges to $g(Q)$ as $X$ converges to $Q$ nontangentially (i.e., $\lim _{x \in \Gamma_{\alpha}(Q) x \rightarrow Q} u(x)=g(Q)$ for every $\alpha>0$, where $\Gamma_{\alpha}(Q)=\{x \in \Omega:|x-Q| \leqslant$ $(1+\alpha) \operatorname{dist}(x, \partial \Omega)\}$, for a.e. $Q(d \sigma) \in \partial \Omega)$.

Proof. We first note that since $\bar{\Omega}$ is compact, we can find $\varphi \in C^{\infty}(\bar{\Omega})$ such that $\varphi(x)>0$ for every $x \in \bar{\Omega}$, and such that $L \varphi(x)<0$ for every $x \in \bar{\Omega}$. (Simply solve $L \psi=-1$ in $\Omega,\left.\psi\right|_{\partial \Omega} \equiv 0$. By the minimum principle, $\psi \geqslant 0$ in $\Omega$, therefore, $\varphi=\psi+1$ satisfies all the required properties.) Consider now $\lambda \varphi$, where $\lambda>0$. Then,

$$
L(\lambda \varphi)+f(x, \lambda \varphi)=\lambda L(\varphi)+O\left((\lambda \varphi)^{p}\right)=\lambda\left(L \varphi+\lambda^{(p-1)} O\left(\varphi^{p}\right)\right)<0
$$

if $\lambda \leqslant \lambda_{0}$, and $\lambda_{0}$ is small enough. Fix such a $\lambda_{0}$ and let $\mu=\lambda_{0} \varphi$. Then, clearly $\mu \in C^{\infty}(\bar{\Omega}), \mu(x)>0$ for every $x \in \bar{\Omega}$, and $L \mu+f(x, \mu)<0$ for $x \in \bar{\Omega}$. Define now

Received by the editors January 22, 1982.

1980 Mathematics Subject Classification. Primary 35J65; Secondary 35J67.

${ }^{1}$ Both authors are supported in part by the N.S.F. The first author is an Alfred P. Sloan Fellow (81-83), the second author is partially supported by a Research Grant from the Graduate School of the University of Minnesota. 
$\varepsilon_{0}=\min _{x \in \bar{\Omega}} \mu(x)$. We will first show that if $0<\varepsilon \leqslant \varepsilon_{0}$, then we can find $u \in C^{2, \alpha}(\bar{\Omega})$, so that $L u+f(x, u)=0$ in $\Omega, u(x)>0$ in $\Omega$ and $\left.u\right|_{\partial \Omega}=\varepsilon$. We first note that, without loss of generality, we can assume that $f(x, u)$ is increasing in $u$. (Substitute $f$ by $f+M u$, and $L$ by $L-M u$, where $M$ is large.) We will assume $f$ to be increasing in the rest of the proof.

Now let $v_{1}$ be the solution to the linear problem

$$
L v_{1}+f(x, \mu)=0,\left.\quad v_{1}\right|_{\partial \Omega}=\varepsilon .
$$

Then, as $f$ is $C^{\alpha}, v_{1} \in C^{2, \alpha}$, moreover, as $\mu \geqslant 0$, and hence $f(x, \mu) \geqslant 0$, the minimum principle shows that $v_{1} \geqslant \varepsilon$ in $\Omega$. In addition, $L\left(v_{1}-\mu\right)=-L \mu-f(x, \mu)>0$ and $\left.\left(v_{1}-\mu\right)\right|_{\partial \Omega} \leqslant 0$. Hence, $v_{1} \leqslant \mu$. Inductively, define $v_{k+1}$ as the solution of

$$
L v_{k+1}+f\left(x, v_{k}\right)=0,\left.\quad v_{k+1}\right|_{\partial \Omega}=\varepsilon .
$$

Then, arguing as above we can check that $\varepsilon \leqslant v_{k+1} \leqslant v_{k} \leqslant \cdots \leqslant \mu$. Now, set $u(x)=\lim _{k \rightarrow \infty} v_{k}(x)$. We first show that $L u=-f(x, u)$ in $\Omega$ in the sense of distributions. In fact, if $\varphi \in C_{0}^{\infty}(\Omega)$, then,

$$
\int_{\Omega}(L u+f(x, u)) \varphi=\int_{\Omega}(u L \varphi+f(x, u) \varphi)=\lim _{k \rightarrow \infty} \int_{\Omega}\left(v_{k} L \varphi+f\left(x, v_{k}\right) \varphi\right)=0,
$$

by dominated convergence. Moreover, it is easy to see that the $v_{k}$ are uniformly bounded in $W^{2, p}(\Omega)$ for every $p, 1 \leqslant p<\infty$. Therefore, $u \in W^{2, p}(\Omega)$, for every $p$, $1 \leqslant p<\infty$, and hence $L u=-f(x, u)$ in the $W^{2, p}$ sense. Since $f \in C^{\alpha}$ it is easy to conclude now that $u \in C^{2, \alpha}(\bar{\Omega})$ is a classical solution of $L u+f(x, u)=0,\left.u\right|_{\partial \Omega}=\varepsilon$.

A similar argument shows the existence of a number $\varepsilon_{0}>0$ so that if $-\varepsilon_{0} \leqslant-\varepsilon<0$, we can find a solution $u \in C^{2, \alpha}(\bar{\Omega})$ to $L u+f(x, u)=0,\left.u\right|_{\partial \Omega}=-\varepsilon$. Also we have $u<-\varepsilon$ in $\Omega$.

We are now ready to prove our theorem. Let $\varepsilon_{0}$ be as above, and $\bar{v}, \underline{v} \in C^{2, \alpha}(\bar{\Omega})$ the solutions of $L \bar{v}+f(x, \bar{v})=0$ (resp. $L \underline{v}+f(x, \underline{v})=0$ ) and $\left.\bar{v}\right|_{\partial \Omega}=\varepsilon_{0}$ (resp. $\left.\left.\underline{v}\right|_{\partial \Omega}=-\varepsilon_{0}\right)$, with $\bar{v} \geqslant \varepsilon_{0}, \underline{v} \leqslant-\varepsilon_{0}$. As before, let $v_{1}$ be a bounded solution of

$$
L v_{1}+f(x, \bar{v})=0, \quad v_{1} l_{\partial \Omega}=g,
$$

in the sense that $v_{1} \in C^{2, \alpha}(\Omega)$, and the boundary values are taken nontangentially a.e. Then the maximum and minimum principle still apply (see for example $[\mathbf{W}]$ ), and we have $\underline{v} \leqslant v_{1} \leqslant \bar{v}$, in $\Omega$. As before, we inductively define $v_{k+1}$ as the bounded solution of the linear problem

$$
L v_{k+1}+f\left(x, v_{k}\right)=0,\left.\quad v_{k+1}\right|_{\partial \Omega}=g,
$$

where the boundary values are taken nontangentially a.e. By the same argument as before, $\underline{v} \leqslant v_{k+1} \leqslant v_{k} \leqslant \cdots \leqslant \bar{v}$. Again, set $u(x)=\lim _{k \rightarrow \infty} v_{k}(x)$. Arguing as before, $L u=-f(x, u)$ in the distribution sense, and also in $W_{\text {loc }}^{2 . p}(\Omega)$, and hence $u \in C^{2, \alpha}(\Omega)$ and $L u+f(x, u)=0$ in $\Omega$ in the classical sense. To see that the boundary values are taken in the desired sense, we argue as follows. Let $v$ be the bounded solution of the linear problem

$$
L v=0 \quad \text { in } \Omega,\left.\quad v\right|_{\partial \Omega}=g,
$$

where the boundary values are taken nontangentially a.e. Let $Z \subset \partial \Omega$ be the set of $Q \in \partial \Omega$ such that the nontangential limit for $v$ at $Q$ does not equal $g(Q)$. By [W], $Z$ 
has surface measure 0 . Let $\varepsilon>0$ be given now, let $Q \in \partial \Omega \backslash Z$, and fix a nontangential approach region $\Gamma_{\alpha}(Q)$. Then, we claim that there exists $\delta>0$, depending only on $\varepsilon, \alpha, \Omega$, but not on $k$ so that

$$
\sup _{x \in \Gamma_{\alpha}(Q) \cap B_{\delta}(Q)}\left|v_{k}(x)-g(Q)\right|<\varepsilon,
$$

where $B_{\delta}(Q)$ is the ball of radius $\delta$ in $\mathbf{R}^{n}$, centered at $Q$. From this claim it is immediate that, for $Q \in \partial \Omega \backslash Z, u(x) \rightarrow g(Q)$ as $x$ converges nontangentially to $Q$. To establish the claim, rewrite $v_{k+1}=v+w_{k}$, where $w_{k}$ is the Green potential for $L$ of $f\left(x, v_{k}\right)$, i.e. $w_{k} \equiv 0$ on $\partial \Omega$, and $L w_{k}=-f\left(x, v_{k}\right)$. Since $\left\|f\left(x, v_{k}\right)\right\|_{\infty} \leqslant M$, where $M$ is independent of $k$, standard elliptic estimates for $w_{k}$ show that $\left\|w_{k}\right\|_{W^{2, p}(\Omega)} \leqslant \tilde{M}$, where $\tilde{M}$ is independent of $k$, and therefore, $\left\|w_{k}\right\|_{C^{\beta}(\bar{\Omega})} \leqslant N, N$ independent of $k$, for any $\beta, 0<\beta<1$. Therefore, as $\left.w_{k}\right|_{\partial \Omega} \equiv 0$, given $\varepsilon>0$, we can choose $\delta>0$, independent of $k$ so that

$$
\sup _{Q \in \partial \Omega} B_{\delta}(Q) \cap \Omega w_{k}(x) \mid \leqslant c \delta^{\beta} \leqslant \frac{\varepsilon}{2} .
$$

Choosing $\delta$ also so that

$$
\sup _{x \in \Gamma_{\alpha}(Q) \cap B_{\delta}(Q)}|v(x)-g(Q)| \leqslant \frac{\varepsilon}{2}
$$

(which is possible by our choice of $Z$ ), our claim follows.

REMARKS. (1) If $g \geqslant 0$, and $g>0$ on a set of positive surface measure of $\partial \Omega$, our construction produces a positive solution in $\Omega$.

(2) If $g \in C(\partial \Omega)$, an easy modification of our argument shows that $u \in C(\bar{\Omega})$, and $\left.u\right|_{\partial \Omega}=g$ at every point. Likewise, if $g \in C^{\beta}(\partial \Omega), 0<\beta<1, u \in C^{\beta}(\bar{\Omega})$.

(3) Our proof also shows that if $Z \subset \partial \Omega$, is a set of 0 surface area for which there exists a bounded function $v$ which is a solution of $L v=0$, and which fails to have nontangential limits at every point $Q$ of $Z$, then we can construct a nonnegative bounded solution $u$ of $L u+f(x, u)=0$, which has the same property.

(4) Arguing as in the proof of the last claim in the proof of Theorem 1, using the results of [W], it is possible to show that if $u$ is any solution of $L u+f(x, u)=0$, which is bounded in $\Omega$, then $u$ has nontangential limits a.e. on $\partial \Omega$.

(5) Using the results in $[\mathbf{J}, \mathbf{K}]$, and the estimates for Green potentials in $[\mathbf{M}]$, it is possible to extend, modifying the proof only slightly, Theorem 1 and Remarks (1), (2), (4) and (5) after it to the case when $\Omega$ is a bounded Lipschitz domain in $\mathbf{R}^{n}$. Remark (3) also holds in this case, provided that in the case $g \in C^{\beta}(\partial \Omega)$, we restrict ourselves to $\beta \leqslant \beta_{0}$, where $\beta_{0}>0$ is a number which depends only on the Lipschitz character of $\partial \Omega$. Also, the smoothness assumptions on the coefficients $a_{i j}(x)$ can be considerably relaxed (for example, it is enough to assume $a_{i j}(x) \in C^{1}\left(\mathbf{R}^{n}\right)$ ).

(6) In the proof of Theorem 1, we have actually proved the following extension of the classical super- and sub-solutions method, which seems to be of some independent interest:

Consider the following Dirichlet problem

$$
L u+f(x, u)=0 \quad \text { in } \Omega,\left.\quad u\right|_{\partial \Omega}=g,
$$


where $\Omega$ is a bounded smooth domain in $\mathbf{R}^{n}, g \in L^{\infty}(\partial \Omega)$ and $f$ is either Lipschitz in $u$ (and Hölder $C^{\alpha}$ in both variables) or increasing in $u$ and $C^{\alpha}$ in $(x, u)$. We say $v$ is a super-( sub-) solution of $(\mathrm{D})$ if

$$
L v+f(x, v) \leqslant(\geqslant) 0,\left.\quad v\right|_{\partial \Omega} \geqslant(\leqslant) g .
$$

Now, we have

Proposition. If $\varphi \in C^{2}(\bar{\Omega})$ is a super-solution of (D) and $\psi \in C^{2}(\bar{\Omega})$ is a sub-solution of (D) with $\varphi \geqslant \psi$, then (D) possesses a solution $u$ with $\varphi \geqslant u \geqslant \psi$.

The following theorem explains the role of $\varepsilon_{0}$ in Theorem 1 .

THEOREM 2. Consider the Dirichlet problem

$$
\Delta u+|u|^{p}=0 \quad \text { in } B,\left.\quad u\right|_{\partial B}=g,
$$

where $B$ is the unit ball in $\mathbf{R}^{n}, p>1, g \in L^{\infty}(\partial B), g \geqslant 0$ and $g \neq 0$. Then, there exists a number $\varepsilon_{0}>0$ such that if $\|g\|_{\infty} \leqslant \varepsilon_{0}$, then $(*)$ has a solution, and such that if

$$
\frac{1}{\sigma(\partial B)} \int_{\partial B} g d \sigma>\varepsilon_{0},
$$

then $(*)$ has no solution.

Proof. First consider the case $g \equiv c>0$, a constant. It is easy to see (from $[\mathbf{G}, \mathbf{N}, \mathbf{N}])$ that a solution of $(*)$ must be radially symmetric. It is then not hard to show that there exists an $\varepsilon^{*}>0$ such that $(*)$ does not have a solution if $c>\varepsilon^{*}$. (For $n>2$, see, for example, p. 260, Theorem 1(ii) in $[\mathbf{J}, \mathbf{L}]$. For the case $n=2$, it may be treated as follows: first find a positive solution $u$ of $(*)$ with $g \equiv 0$, then consider $v(r)=\lambda^{2 /(p-1)} u(\lambda r)$ for $0<\lambda \leqslant 1, r \leqslant 1$. This is a one-parameter family of solutions of $(*)$ with $g_{\lambda} \equiv \lambda^{2 /(p-1)} u(\lambda)$. As $\lambda \rightarrow 0, g_{\lambda} \rightarrow 0$; also, as $\lambda \rightarrow 1, g_{\lambda} \rightarrow 0$. Thus, we may let $\varepsilon^{*}=\max _{\lambda \in(0,1)} g_{\lambda}$.)

Since it is easy to show (*) has a positive solution for some $g \equiv c>0$ (using the same rescaling idea as above), the argument in Theorem 1 shows there exists an $\varepsilon_{0}>0$ such that $(*)$ possesses a solution if $g \equiv c \leqslant \varepsilon_{0}$ but not otherwise.

The proof of Theorem 1 furnishes existence when $\|g\|_{\infty} \leqslant \varepsilon_{0}$. For nonexistence when $1 / \sigma(\partial B) \int_{\partial B} g d \sigma>\varepsilon_{0}$, we argue by contradiction. If $(*)$ had a solution $u$ in this case, we set $\bar{u}(r)=$ average of $u$ on the sphere of radius $r$. Then, by a standard argument (see for example $[\mathbf{N}]$ ), $\Delta \bar{u}+\bar{u}^{p} \leqslant 0,\left.\bar{u}\right|_{\partial B}=1 / \sigma(\partial B) \int_{\partial B} g>\varepsilon_{0}$, which, arguing as in Theorem 1, contradicts the results above.

\section{REFERENCES}

[G, N, N] B. Gidas, W.-M. Ni and L. Nirenberg, Symmetry and related properties via the maximum principle, Comm. Math. Phys. 68 (1979), 209-243.

[J, K] D. S. Jerison and C. E. Kenig, The Dirichlet problem in non-smooth domains, Ann. of Math. (2) 113 (1981), 367-382.

[J,L] D. D. Joseph and T. S. Lundgren, Quasilinear Dirichlet problems driven by positive sources, Arch. Rational Mech. Anal. 49 (1973), 241-269.

[K, W] J. L. Kazdan and F. W. Warner, Curvature functions for open 2-manifolds, Ann. of Math. (2) 99 (1974), 203-219. 
[M] C. B. Morrey, Second order elliptic equations in several variables and Hölder continuity, Math. Z. 72 (1959), 146-164.

[N] W.-M. Ni, On the elliptic equation $\Delta u+K(x) u^{(n+2) /(u-2)}=0$, its generalizations and applications to geometri, Indiana Univ. Math. J. 31 (1982), 493-529.

[S] D. Sattinger, Topics in stability and bifurcation theory, Lecture Notes in Math., vol. 309, SpringerVerlag. Berlin and New York, 1973.

[W] K. O. Widman, On the houndary hehavior of solutions to a class of elliptic partial differential equations, Ark. Mat. 6 (1966), 485-533.

School of Mathematics, University of Minnesota, Minneapolis, Minnesota 55455 